\title{
Hardware Imitation of the Varying Bioelectrical Impedance for Testing the Device for Measurement of the Impedance of Human Trunk by Using a JFET
}

\author{
Margus Metshein \\ Th. J. Seebeck Dept. of Electronics \\ Tallinn University of Technology \\ Estonia, Tallinn \\ margus.metshein@ttu.ee
}

\begin{abstract}
In this paper an approach of imitating the periodically varying electrical bioimpedance of human trunk in case of breathing by using a field effect transistor is presented. The principles of creating a simulation model and preparing a prototype of electronic measurement device, are described. The experiments are presented and the results are compared concerning the change of channel resistance of the field effect transistor and change of real part of impedance of trunk, caused by breathing. The problems of high frequency capacitive measurements and the impact on the design of electronic measurement device are discussed. The results are showing the possibility of using a field effect transistor for imitating breathing. This is a most suitable way of simulation because of the relatively high operating frequency of impedance measurement device needed in the case of using the capacitive electrodes.
\end{abstract}

Keywords- capacitive electrodes; impedance measurement; JFETs; wearable sensors

\section{INTRODUCTION}

Monitoring the condition of human body has gained plenty of attention in the last decade. The main reason for this are the development of technology, the ever-increasing availability of more and more skillful personal multimedia devices and the recommendations from health organizations for regular physical training.

The measuring of the electrical bioimpedance (EBI) proposes a possibility to follow the changes taking place in biological media. The idea of EBI measurement is to excite human body with small electrical current or voltage and measure the response [1]. In general, the vital processes of breathing and heart rate (HR) are of main interest of current paper. Both of these processes are periodically repeating and can be followed by measuring the EBI of the body. There are several attempts described, to prepare a portable measurement devices for implementing the task of measuring breathing and HR of human by the means of EBI [2]-[4].

In this paper, an approach of imitating the changing impedance of human trunk that is caused by breathing by a field effect transistor (FET), is described. The FET is used as voltage controllable resistor in its ohmic operating region. In this region, the channel resistance $\left(\mathrm{R}_{\mathrm{DS}}\right)$ of a FET can be changed by varying the gate-to-source voltage $\left(\mathrm{V}_{\mathrm{GS}}\right)$.

In the published works are variety of approaches described for imitating the impedance of human body, for example: electronic module with controllable impedance [5], mechanical phantom that imitates breathing [6] etc. In current paper, a straightforward approach of imitating the variable impedance of trunk by a single FET is described.

The results of the measurements accomplished using the FET imitator are compared with the results of the measurements of the resistance of the real human trunk. For implementing this task, a wearable measurement device in the form of shirt was used. The shirt was covered by large plate surface electrodes which were used in capacitive connection to the trunk.

The simulation model and prototype of the device were prepared and the measurements were done to investigate the possibility of using a FET to imitate breathing. The results were analyzed and conclusions made. All described experiments were done with the goal of developing a wearable measurement device for determining breathing and HR of human.

\section{DeVICES AND PROPERTIES}

\section{A. Principle and the Realization of the Prototype of} Custom Made Electronic Measurement Device

The idea of the impedance measurement device includes the exciting of the object with a square wave signal and measuring of the real part (ReZ) of the total impedance (modulus $|\mathrm{Z}|$ ) by using two-electrode configuration [7].

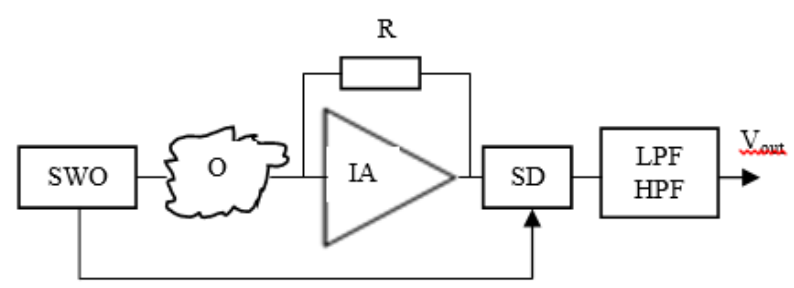

Figure 1. Block diagram of the device for EBI measurements 
The basic principle of the EBI measurement device can be seen from the block diagram in Fig. 1, which has been shown already in [7]. This device consists of the square wave oscillator (SWO), an object of interest (O), the inverting operational amplifier (IA) with feedback resistor $\mathrm{R}$ in its feedback path, the synchronous detector (SD) and the low- and highpass filters (LPF and HPF).

\section{B. Utilized Laboratory Devices and Properties}

As power supply, E3631A of Agilent Technologies and as signal oscillator HF2IS impedance spectroscope of Zurich Instruments were used. For electrical measurements, digital multimeter KEW1012 of Kyoritsu was used. In the chosen measurement ranges KEW1012 proposes the accuracy of $\pm 0.5 \%$ with reading error of \pm 2 digits for voltage and $\pm 1.2 \%$ with reading error of \pm 3 digits for current measurements.

\section{Properties of FET in the Case of Operating as Voltage Controlled Resistor}

It is known that in certain condition, the FET can operate as a voltage controlled resistor - this means that the voltage drop on the channel $\left(\mathrm{V}_{\mathrm{DS}}\right)$ is nearly linearly dependent on current through the channel $\left(\mathrm{I}_{\mathrm{D}}\right)$ (that means $\mathrm{R}=\mathrm{V}_{\mathrm{DS}} / \mathrm{I}_{\mathrm{D}}$ is valid) and resistance $\mathrm{R}$ is a function of $\mathrm{V}_{\mathrm{GS}}$ (Fig. 2).

The $I_{D}$ is maximum ( $I_{D S S}$ ) and $R_{D S}$ is minimum when the value of $\mathrm{V}_{\mathrm{GS}}$ is 0 . If $\mathrm{V}_{\mathrm{GS}}$ is heighten, $\mathrm{R}_{\mathrm{DS}}$ will increase. If $I_{D}$ is reduced to zero, then $R_{D S}$ has reached its maximum resistance - this is called pinch-off voltage $\left(\mathrm{V}_{\mathrm{GS}(\mathrm{off})}\right)$.

Here the dashed parabolic line separates the region in which FET acts quite like resistor (so called ohmic or linear region) and the region where the value of $I_{D}$ is no more rising if $\mathrm{V}_{\mathrm{DS}}$ is increasing (saturation region). The region of linear enough operation is, of course, much more limited than ohmic region.

For the experiments, a junction FET (JFET) of type BF256B of Fairchild Semiconductor was chosen. This proposes the values of $\mathrm{V}_{\mathrm{GS}}$ in interval of $-0.5 \ldots$ $-7.5 \mathrm{~V}$ (in the case of $\mathrm{V}_{\mathrm{DS}}$ is equal to $15 \mathrm{~V}$ and $\mathrm{I}_{\mathrm{D}}$ is $200 \mu \mathrm{A}$ ) and $\mathrm{I}_{\mathrm{DSS}}$ in the interval of $6 \ldots 13 \mathrm{~mA}$.

\section{Methods And Measurement SETuP}

\section{A. Setup for the Measuring the $R_{D S}$ of BF256B in} Simulation Model and by Using the Custom Made Electronic Measurement Device

A model of the EBI measurement device in simulation environment Multisim 13.0 was created for the characterization. To represent the change of ReZ of human trunk in the model during breathing, a JFET with marking $V T$ was used as a voltage controlled resistor (Fig. 3). The value of resistance for the fixed resistor $R_{2}$ was chosen to be $100 \Omega$, representing the continuous resistance of nonchanging matter of human body. JFET was intended to represent the changing impedance of trunk that is caused by breathing. For finding the maximum and minimum values of $\mathrm{R}_{\mathrm{DS}}$ of JFET, a DC voltage supply, for providing constant values of -1.1 volts and -0.5 volts, was added to the circuit, representing $\mathrm{V}_{\mathrm{GS}}$.

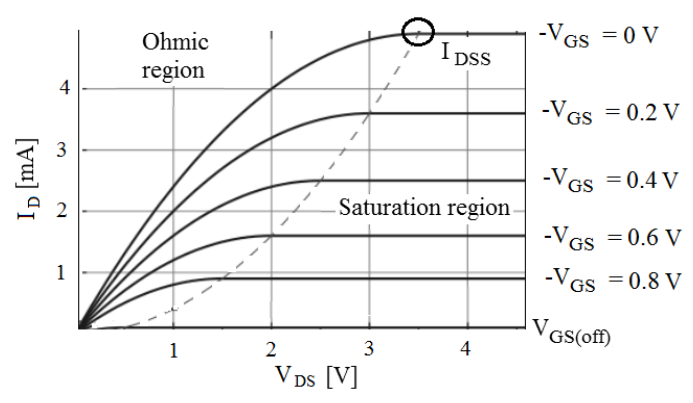

Figure 2. Principal characteristics of N-channel JFET

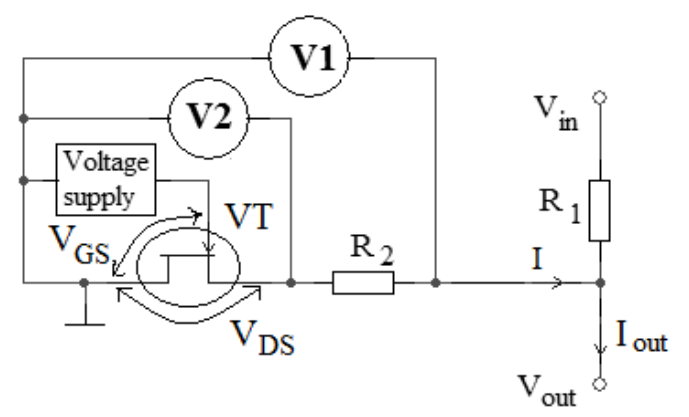

Figure 3. Circuit setup for measuring the values of $V_{D S}$ and $I_{D}$ of $\mathrm{BF} 256 \mathrm{~B}$ in simulation model and by the custom made electronic measurement device for calculating $R_{D S}$

Input $\mathrm{V}_{\text {in }}$ was provided by signal oscillator, supplying a square wave rail-to-rail signal with amplitude of $3 \mathrm{~V}_{\mathrm{pp}}$ and frequency of $10 \mathrm{MHz}$. Resistor $R_{1}$ was forming a voltage divider with resistor $R_{2}$. $\mathrm{V}_{\text {out }}$ forms the input for the op-amp in the next stage of the device - IA (Fig. 1).

In case of measuring the $\mathrm{V}_{\mathrm{DS}}$ and $\mathrm{I}_{\mathrm{D}}$ for JFET $V T$ by using the prepared electronic measurement device, DC voltage supply was set to values $-1.1 \mathrm{~V}$ and $-0.5 \mathrm{~V}$ to represent $\mathrm{V}_{\mathrm{GS}}$. The readings of voltmeters $V 1$ and $V 2$ and the value of I were saved for different values of $\mathrm{V}_{\mathrm{GS}}$ in the interval of $-1.1 \ldots-0.5 \mathrm{~V}$.

\section{B. Setup for Measuring the ReZ of Human Trunk}

To measure ReZ of human trunk, a custom made electrode shirt was used, described in [8]. The device was composed in a way that large wet plate surface electrodes of dimensions $80 \mathrm{~mm}$ x $170 \mathrm{~mm}$ were attached onto the outer surface of a cotton shirt and connected in desired configuration. The thickness of cotton shirt was about $1 \mathrm{~mm}$. For the experiments, described in the current paper, the same setup was used, which is described in [7]. The placement of electrodes, seen in Fig. 4, is expected to cover the area of trunk, setting lungs between the electrodes.

An experiment was set up to measure the voltage and current that flows through trunk of human (Fig. 4). An input signal $V_{\text {in }}$ of rail-to-rail signal amplitude of $3 \mathrm{~V}$ and frequency of $10 \mathrm{MHz}$ was applied to the object. Voltage U (voltmeter $V$ ) and current I were measured in two conditions:

- holding the breath while lungs are filled with air,

- holding the breath while air is blown out of lungs. 


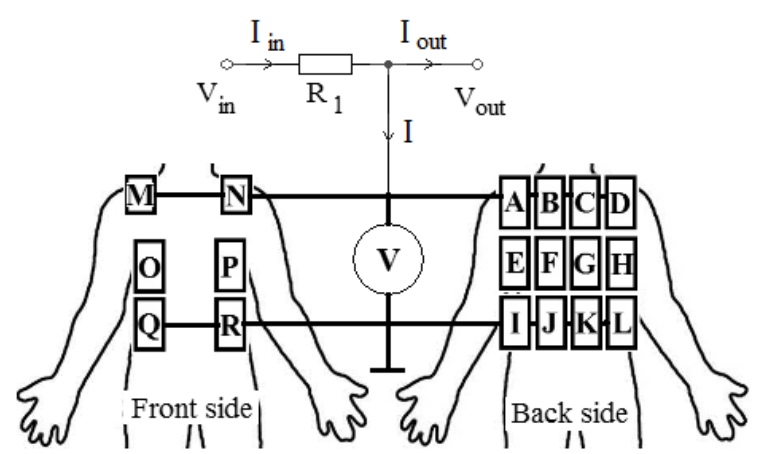

Figure 4. Circuit setup for determining $\mathrm{R}_{\mathrm{DS}}$ of human trunk with custom made electronic measurement device when lungs are filled with air and vice versa

\section{MEASUREMENT Results}

\section{A. Results of Characterization of $R_{D S}$ of BF $256 B$ in Simulation Model}

For characterization of the JFET as a voltage controlled resistor in the simulation model, the values of $V_{D S}$ and $I_{D}$ were measured. The measurements were done with and without additional resistor $R_{2}-$ in the case of $V 2, R_{2}$ was short-circuit. Single measurement was carried out in both setups in both values of $V_{\mathrm{GS}}$ and no accuracy is shown because the ideal conditions were assumed to occur in the model. The values $R_{D S V 1}$ and $R_{D S V}$ for both values of $V_{G S}$ were calculated by using Ohm's law - the results can be seen in Table I.

TABLE I. RESULTS OF MEASUREMENTS OF PARAMATERS OF BF256B IN THE SIMULATION MODEL

\begin{tabular}{|c|c|c|}
\hline Variable & $\begin{array}{c}\text { Value when } \\
\text { V }_{\text {GS }}=\mathbf{- 1 . 1} \mathbf{~ V}\end{array}$ & $\begin{array}{c}\text { Value when } \\
\text { V }_{\text {GS }}=\mathbf{- 0 . 5} \mathbf{~ V}\end{array}$ \\
\hline $\mathrm{V} 1$ & $1084 \mathrm{mV}$ & $789 \mathrm{mV}$ \\
\hline $\mathrm{V} 2$ & $904 \mathrm{mV}$ & $581 \mathrm{mV}$ \\
\hline $\mathrm{I}$ & $1.80 \mathrm{~mA}$ & $2.10 \mathrm{~mA}$ \\
\hline $\mathrm{R}_{\mathrm{DSV} 1}$ & $602 \Omega$ & $376 \Omega$ \\
\hline $\mathrm{R}_{\mathrm{DSV} 2}$ & $502 \Omega$ & $276 \Omega$ \\
\hline
\end{tabular}
by:

The difference of the values of $R_{D S}$ was calculated

$$
R_{\triangle J F E T}=R_{D S(-1.1)}-R_{D S(-0.5)}
$$

where:

$\mathrm{R}_{\triangle \mathrm{JFET}}$ - change of the value of $\mathrm{R}_{\mathrm{DS}}(\Omega)$;

$\mathrm{R}_{\mathrm{DS}(-1.1)}$ - value of $\mathrm{R}_{\mathrm{DS}}$ when $\mathrm{V}_{\mathrm{GS}}=-1.1 \mathrm{~V}(\Omega)$;

$\mathrm{R}_{\mathrm{DS}(-0.5)}$ - value of $\mathrm{R}_{\mathrm{DS}}$ when $\mathrm{V}_{\mathrm{GS}}=-0.5 \mathrm{~V}(\Omega)$.

The value of $R_{\triangle \text { JFET }}$ in both cases was $226 \Omega$ and the difference between the values of $R_{D S}$ in both values of $\mathrm{V}_{\mathrm{GS}}$ was exactly $100 \Omega$ - the value of additional resistor $\mathrm{R}_{2}$.

For evaluating the behavior of JFET, the characteristics of $\mathrm{R}_{\mathrm{DS}} \mathrm{vs}$. $\mathrm{V}_{\mathrm{GS}}$ were drawn in setup where the resistor $R_{2}$ was omitted, for both measurements: in simulation model and for electronic measurement device (Fig. 5). In the same figure, the change of $R_{D S}$ in chosen operating area $V_{O A}$ $\left(\mathrm{V}_{\mathrm{GS}}=-1.1 \ldots-0.5 \mathrm{~V}\right)$ can be seen

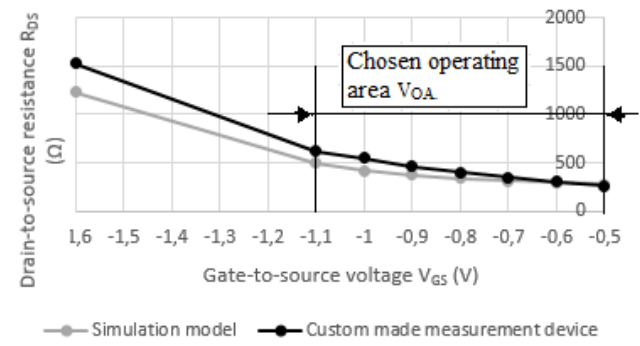

Figure 5. Characteristics of $\mathrm{V}_{\mathrm{GS}}$ vs. $\mathrm{R}_{\mathrm{DS}}$ of $\mathrm{BF} 256 \mathrm{~B}$ in simulation model

B. Results of Characterization of $R_{D S}$ of BF $256 B$ by Using the Custom Made Electronic Measurement Device

For characterization of the voltage controlled FET by electronic measurement device, the values of $\mathrm{V}_{\mathrm{DS}}$ and $\mathrm{I}_{\mathrm{D}}$ were measured by using above described laboratory instruments. Several measurement cycles were carried out with both values of $\mathrm{V}_{\mathrm{GS}}$ : $-1.1 \mathrm{~V}$ and $0.5 \mathrm{~V}$. The values of $\mathrm{R}_{\mathrm{DSV} 1}$ and $\mathrm{R}_{\mathrm{DSV} 2}$ (Fig. 3) for both values of $\mathrm{V}_{\mathrm{GS}}$ were calculated by using Ohm's law. The results can be seen in Table II, where the median values are shown together with accuracy.

TABLE II. RESULTS OF MEASUREMENTS OF PARAMETERS OF BF256B BY USING THE CUSTOM MADE ELECTRONIC MEASUREMENT DEVICE

\begin{tabular}{|c|c|c|}
\hline Variable & $\begin{array}{c}\text { Value when } \\
\text { V }_{\text {GS }}=\mathbf{- 1 . 1} \mathbf{~ V}\end{array}$ & $\begin{array}{c}\text { Value when } \\
\mathbf{V}_{\text {GS }}=\mathbf{- 0 . 5} \mathbf{~ V}\end{array}$ \\
\hline $\mathrm{V} 1$ & $25.2 \pm 0.13 \mathrm{mV}$ & $3.50 \pm 0.02 \mathrm{mV}$ \\
\hline $\mathrm{V} 2$ & $41.4 \pm 0.21 \mathrm{mV}$ & $15.8 \pm 0.08 \mathrm{mV}$ \\
\hline $\mathrm{A}$ & $66.5 \pm 0.80 \mu \mathrm{A}$ & $59.7 \pm 0.72 \mu \mathrm{A}$ \\
\hline $\mathrm{R}_{\mathrm{DSV} 1}$ & $379 \pm 2.58 \Omega$ & $58.6 \pm 0.40 \Omega$ \\
\hline $\mathrm{R}_{\mathrm{DSV} 2}$ & $623 \pm 3.94 \Omega$ & $265 \pm 1.53 \Omega$ \\
\hline
\end{tabular}

The values of $R_{D S}$ were calculated by using (1). The intervals of changes of the value of $\mathrm{R}_{\mathrm{DS}}$ are:

- $\quad$ with additional resistor $\mathrm{R}_{2}, \mathrm{R}_{\mathrm{DSV} 1}=320.4 \Omega$;

- $\quad$ without additional resistor $\mathrm{R}_{2}, \mathrm{R}_{\mathrm{DSV} 2}=358 \Omega$.

\section{Results of Measurements of ReZ of Human Trunk}

Several measurement were carried out to measure voltage $(\mathrm{V})$ by using large plate surface electrodes that were placed on human trunk and the current (I) that flows through trunk in two conditions - lungs filled with air (inhale) and air breathe out (exhale) (Fig. 4). The values of ReZ, which can be marked as $R_{\text {exhale }}$ and

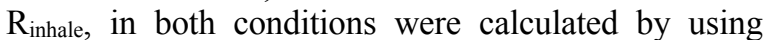
Ohm's law. The results can be seen in Table III, where the median values are shown together with accuracy.

TABLE III. RESULTS OF MEASUREMENTS OF PARAMETERS OF HuMAN TRUNK BY USING THE CUSTOM MADE ELECTRONIC MEASUREMENT DEVICE

\begin{tabular}{|c|c|c|}
\hline Variable & Exhale & Inhale \\
\hline $\mathrm{V}$ & $98.83 \pm 0.496 \mathrm{mV}$ & $100.0 \pm 0.502 \mathrm{mV}$ \\
\hline $\mathrm{A}$ & $50.63 \pm 0.611 \mu \mathrm{A}$ & $48.43 \pm 0.584 \mu \mathrm{A}$ \\
\hline $\mathrm{R}$ & $1952 \pm 13.93 \Omega$ & $2065 \pm 14.55 \Omega$ \\
\hline
\end{tabular}

The differences of the values of ReZ between the moment when lungs are filled with air and the moment when air is breathe out, can be calculated by: 


$$
R_{\Delta \text { trunk }}=R_{\text {inhale }}-R_{\text {exhale }},
$$

where:

$\mathrm{R}_{\Delta \text { trunk }}$ - change of the value of $\operatorname{ReZ}(\Omega)$;

$\mathrm{R}_{\text {inhale }}$ - value of ReZ when lungs filled with air $(\Omega)$;

$\mathrm{R}_{\text {exhale }}$ - value of ReZ when air breathe out $(\Omega)$.

The results show that the interval of change of ReZ, caused by in- and exhalation is $113 \Omega$.

\section{DISCUSSION}

The results of the described experiments propose a possibility to compare the interval of change of $R_{D S}$ of JFET, caused by different values of $V_{G S}$ and interval of change of ReZ of human trunk, caused by in- and exhalation. The characteristics of JFET are expected to accord to the data to predict the ability of the developed prototype of the electronic measurement device for granting the access to breathing of human by measuring $\mathrm{ReZ}$ of trunk.

The results of the characterization of JFET in simulation model show the possibility of measuring the variations of $R_{D S}$. Thereby, in ideal conditions, the resistor $R_{2}$ does not have affection to the result of the difference of the value of $R_{D S}$.

The measurements of parameters of chosen JFET by using the electronic measurement device, show the affection of external interferences and the influence of relatively high frequency input signal. The difference of the values of $R_{\mathrm{DS}}$ with and without $R_{2}$ do not hold the same value. Value of $R_{D S}$ in case of setup without $R_{2}$ has increased when compared to the measurements in simulation model.

The measurements of ReZ of human trunk show the accessibility of in- and exhalation. When lungs are filled with air, ReZ of trunk is assumed to increase because of the increase of nonconducting matter in object. When air is breathe out, ReZ is assumed to decrease because of the decrease of the same matter. The interval of change of the value of ReZ of trunk between these conditions is about three times narrower when comparing to $\mathrm{R}_{\mathrm{DS}}$ of JFET. The measured values of ReZ give hope that breathing can be followed by measuring ReZ of trunk with presented device.

The interval of change of ReZ of trunk is small enough to be prone to interferences. The latter fact is illustrated in [7]. A short study was made during the experiments - measurement of the same variables in two different positions of human: standing and sitting on a chair with leaning onto the back. In first case the shirt is hanging freely and in second case the electrodes on the back are pressed against the skin. The differences between the calculated values of ReZ were hundreds of ohms.

The reason can be found at the properties of capacitive connection, which resembles the principle of capacitor. Basically, the electrode and the epidermis with body tissues are forming the structure of capacitor. The changing distance between the "plates" and the electrode overlap changes the value of capacitance.

\section{CONCLUSIONS AND FUTURE WORK}

Utilization of a JFET for simulating the respiration show the possibility of using this kind of approach for characterization electronic devices that measure impedance of living organisms. This constitutes a robust solution for imitating the variable processes that take place in human body - like breathing and HR.

The comparison of the even measuring experiment in simulation model and with electronic measurement device show the difference between the cases of ideal conditions and in real experiments. The properties of real devices may vary and affect the results.

The experimental capacitive measurement in the case of a real object confirm this fact numerically, presenting the difference in measured ReZ when lungs are filled with air and vice versa. Besides, the usage of HF excitation signal and capacitive connection to the object sets the result prone to interferences.

Future work will be implemented during two parallel research: through the characterization of the electronic measurement device; through the design considerations of the electrode shirt and the incorporation of interconnections. The usage of a FET for imitating the processes of breathing and HR gives an option for testing and evaluating these devices.

\section{ACKNOWLEDGMENT}

This research was supported by the European Union through the European Regional Development Funding in the frames of the research center CEBE and by Estonian Research Council (IUT19-11-2014).

\section{REFERENCES}

[1] S. Grimnes and Ø. G. Martinsen, Bioimpedance \& Bioelectricity Basics (Book style), London, Great Britain: Academic, 2008. p. 350.

[2] G. Panfili, L. Piccini, L. Maggi, S. Parini, G. Andreoni, ”A wearable device for continuous monitoring of heart mechanical function based on impedance cardiography", In Proc. of the 28th IEEE EMBS Annual In. Conf., NY, US, Aug. 2006, pp. 5986-5971.

[3] K. Kohli, et al. "Prototype development of an electrical impedance based simultaneous respiratory and cardiac monitoring system for gated radiotherapy" BioMedical Engineering OnLine, 2014, 13:144.

[4] F. Villa, A. Magnini, G. Merati, P. Castiglioni, "Feasibility of long-term monitoring of multifrequency and multisegment body impedance by portable devices", IEEE Trans. on Biomed. Eng. vol. 61, no. 6, June, 2014, pp. 1877-1886.

[5] M. Młyńczak, K. Pariaszewska, W. Niewiadomski, G. Cybul, "Design and construction of the artificial patient module for testing bioimpedance measuring devices", in Proc. of SPIE, vol. 8903, 890314, Oct. 2013, pp. 1-9.

[6] M. J. Fitzpatrick et al. "A novel platform simulating irregular motion to enhance assessment of respiration-correlated radiation therapy procedures", J. of Appl. Clinical Med. Phys., Vol. 6(1), Winter 2005, pp. 13-21.

[7] M. Metshein, T. Parve, "Electrical bioimpedance based monitoring of cardiac and respiratory activity: positioning of capacitive electrodes for wearable human monitoring device", Int. J. of Bio-electromagnetism, 17 (2), pp. 64-74.

[8] M. Metshein, "A device for measuring the electrical bioimpedance with variety of electrode placements for monitoring the breathing and heart rate", in Proc. of 26th Irish Signals and Systems Conference (ISSC), IEEE, Carlow, Ireland, June, 2015, pp. 1-4. 